\title{
THERMAL 3D MAPPING FOR OBJECT DETECTION IN DYNAMIC SCENES
}

\author{
Martin Weinmann ${ }^{a}$, Jens Leitloff ${ }^{a}$, Ludwig Hoegner $^{b}$, Boris Jutzi $^{a}$, Uwe Stilla $^{b}$ and Stefan Hinz ${ }^{a}$ \\ ${ }^{a}$ Institute of Photogrammetry and Remote Sensing, Karlsruhe Institute of Technology (KIT) \\ \{martin.weinmann, jens.leitloff, boris.jutzi, stefan.hinz\}@kit.edu \\ ${ }^{b}$ Photogrammetry and Remote Sensing, Technische Universität München (TUM) \\ ludwig.hoegner@bv.tum.de, stilla@tum.de
}

Commission I, WG I/2

KEY WORDS: Multisensor, point cloud, thermal imaging, 3D mapping, dynamic, object detection

\begin{abstract}
:
The automatic analysis of 3D point clouds has become a crucial task in photogrammetry, remote sensing and computer vision. Whereas modern range cameras simultaneously provide both range and intensity images with high frame rates, other devices can be used to obtain further information which could be quite valuable for tasks such as object detection or scene interpretation. In particular thermal information offers many advantages, since people can easily be detected as heat sources in typical indoor or outdoor environments and, furthermore, a variety of concealed objects such as heating pipes as well as structural properties such as defects in isolation may be observed. In this paper, we focus on thermal 3D mapping which allows to observe the evolution of a dynamic 3D scene over time. We present a fully automatic methodology consisting of four successive steps: (i) a radiometric correction, (ii) a geometric calibration, (iii) a robust approach for detecting reliable feature correspondences and (iv) a co-registration of 3D point cloud data and thermal information via a RANSAC-based $\mathrm{EP} n \mathrm{P}$ scheme. For an indoor scene, we demonstrate that our methodology outperforms other recent approaches in terms of both accuracy and applicability. We additionally show that efficient straightforward techniques allow a categorization according to background, people, passive scene manipulation and active scene manipulation.
\end{abstract}

\section{INTRODUCTION}

The automated description and analysis of static and dynamic 3D scenes represents a topic of major interest in photogrammetry, remote sensing and computer vision. Due to the recent technological advancements, a variety of devices is currently available which can be used for acquiring different types of information such as color, temperature or spatial 3D geometry. Thus, the use of different devices on a common sensor platform allows to collect multidimensional spatial data.

In particular those devices delivering complementary types of information offer a high potential for numerous applications. Modern range cameras such as Microsoft Kinect, PMD[vision] CamCube 2.0 or MESA Imaging SR4000 simultaneously provide geometric information as well as radiometric information in form of range and intensity images, and they are also applicable for adequately capturing dynamic scenes. Whereas the radiometric information is typically represented as color or gray-valued images, other devices such as thermal cameras offer to capture complementary information which can be helpful for describing and analyzing the observed scene and its evolution over time.

A fusion of intensity and thermal information for instance seems desirable, since the respective images reveal a very different behavior:

- Standard intensity images (i.e. color or gray-valued images) typically represent information of the visual domain and thus radiometric surface properties of observed objects. This information may strongly depend on the material and reflectance behavior of respective objects as well as the relative geometric orientation of the surface to the camera.

- Thermal infrared images represent thermal radiation in the infrared spectrum. This radiation is emitted by objects in the scene and not visible in the visual domain. Consequently, the thermal infrared images allow a different look on objects and the extraction of additional information like temperature and different materials of observed objects.

Accordingly, objects visible in the visual domain may be invisible in the infrared domain if they have the same temperature and emissivity coefficient as the respective background. In contrast, in infrared images, even further objects below the surface of an object may be visible which certainly remain invisible in the visual domain. Note that two different materials with the same temperature can appear with different intensity if they have a significantly different emissivity coefficient. Interestingly, two objects with different temperature and emissivity coefficient can even coincidentally appear with very similar intensity in thermal images. As a consequence, a fusion of intensity images and thermal infrared images can reveal information which may not be present in either intensity images or thermal infrared images (Chen and Leung, 2009; Bai et al., 2011). This is for instance of special interest for enhancing contrast in environments of poor visibility or inadequate illumination (Liu and Laganière, 2007), for target detection (Yao and Sekmen, 2008) or for concealed weapon detection (Xue et al., 2002). More importantly, however, infrared thermography allows for building diagnostics (Balaras and Argiriou, 2002) which, due to current attempts for saving energy, has become a research topic itself.

Whereas the fusion of intensity images and thermal infrared images provides different types of information, it does not account for the respective spatial dimensions. Recent technological advancements however allow a thermal 3D mapping in terms of projecting the captured image data onto $3 \mathrm{D}$ point clouds acquired with a terrestrial laser scanner, mobile laser scanner or range camera. The metric information, in turn, allows the quantification of thermal studies if the point clouds are texturized with thermal information (Lagüela et al., 2011b). Especially in building 
observation (Hoegner et al., 2007a; Iwaszczuk et al., 2011), the joint representation of building façades and thermal information allows a reconstruction of the surface temperature and thus a look into the interior behavior of a wall. Valuable insights about different materials, heating pipes and leakages can easily be obtained and added as semantic or geometric information to the respective building model.

In order to capture co-registered intensity information, thermal information and spatial 3D geometry, the combination of data captured with a terrestrial laser scanner and images acquired by a bi-camera system, i.e. a system consisting of an optical camera and a thermal camera, has recently been proposed (Alba et al., 2011). Furthermore, a robot equipped with a 3D laser scanner, a thermal camera and a digital camera has been presented which is capable to generate a precise $3 \mathrm{D}$ model showing the heat distribution in the scene (Borrmann et al., 2012a; Borrmann et al., 2012b). The additional color information can for instance be used for identifying heat sources or for obtaining photo-realistic 3D models. Focusing on the acquisition of dynamic scenes, respective systems addressing thermal 3D mapping involve range cameras such as RGB-D cameras or Time-of-Flight (ToF) cameras. For instance, the use of a low-cost RGB-D camera in combination with a thermal camera has recently been proposed for acquiring dense 3D models of environments with both appearance and temperature information (Vidas et al., 2013). Involving a Timeof-Flight camera, an approach for gaining infrared-textured 3D models has been proposed (Weinmann et al., 2012) which additionally focuses on sharpening the blurry thermal information by exploiting the high quality of intensity information acquired with the range camera.

In this paper, we focus on thermal 3D mapping which allows to observe the evolution of a dynamic 3D scene over time. The main contribution presented in our paper is a fully automatic methodology for thermal 3D mapping which involves

- a geometric calibration of both the range camera and the thermal camera based on a common strategy,

- a robust approach for detecting 2D/2D feature correspondences via shape-based matching,

- a removal of unreliable 2D/2D feature correspondences by considering the reliability of the respective range information, and

- a novel approach exploiting 3D/2D feature correspondences for the co-registration of 3D point cloud data and thermal information.

After reflecting the related work in Section 2, we provide a detailed explanation of the different steps of our new methodology in Section 3. For an experimental setup described in Section 4, we present the derived results in Section 5 and discuss these with respect to different aspects in Section 6. Finally, Section 7 contains conclusions as well as ideas for future work.

\section{RELATED WORK}

For thermal 3D mapping involving a range camera, respective 3D coordinates are available for each pixel of the intensity image. Accordingly, feature correspondences have to be derived either between standard intensity images (i.e. color or gray-valued images) and thermal infrared images (Section 2.1), or between 3D point clouds and thermal infrared images (Section 2.2). For the sake of completeness, we also briefly reflect approaches generating 3D point clouds from thermal infrared images (Section 2.3).

\subsection{Co-Registration of Intensity and Thermal IR Images}

One category of approaches directly leads to basic image processing in terms of image registration (Zitová and Flusser, 2003). Once the respective transformation model has been estimated, the thermal infrared image can be warped onto the intensity image of the range camera, and thus also be projected to 3D space by forward projection according to the respective $3 \mathrm{D}$ information.

Among the approaches for image registration, the feature-based approaches are most widely spread. In general, we may consider any feature present in an image as visual feature. More specifically, according to a recent survey on visual features (Weinmann, 2013), different types of visual features can be categorized, where texture, shape and local features are the most prominent types due to their applicability for numerous applications such as image registration, data retrieval, scene analysis and the autonomous navigation of aerial and ground vehicles. Typically, approaches for feature extraction and matching are tailored for images acquired by either identical or similar sensors from various positions. However, a matching between different image domains e.g. a co-registration of satellite imagery and LiDAR intensity images (Toth et al., 2011) - can be quite challenging as the respective images may have very different characteristics due to which many standard approaches for deriving feature correspondences tend to fail.

In particular, an automatic matching between the visual domain and the thermal domain still remains challenging. Intensity images in the visual domain often provide abrupt changes of properties like intensity or texture at the edges of objects in the observed scene. In thermal infrared images, however, we might face challenges arising from $(i)$ the low geometric resolution compared to classical optical camera systems and (ii) the fact that features such as lines or contours do not show strong edges, but rather appear blurred. As a consequence, even powerful standard methods for deriving feature correspondences, e.g. the Scale Invariant Feature Transform (SIFT) (Lowe, 1999; Lowe, 2004), are not applicable for automatically detecting feature correspondences between the visual domain and the thermal domain.

For registration of image data representing information in these different spectral bands, different approaches have been proposed such as a segment-based approach (Coiras et al., 2000) or an approach involving normalized mutual information (Park et al., 2008). Furthermore, approaches for generating textured $3 \mathrm{D}$ point clouds often rely on a matching between images followed by forward projection to 3D space. For flat building façades and almost planar scenes, the transformation model of a homography (Hartley and Zisserman, 2008) can be applied. Such a transformation may for instance be exploited for mapping thermal IR information on existing building models (Hoegner et al., 2007b) or thermal 3D mapping involving a range camera (Weinmann et al., 2012). The latter approach relies on deriving feature correspondences between the respective images via shape-based matching, a respective image registration and a subsequent forward projection to 3D space. Considering mutual information between images has also been proposed for mapping multispectral texture information onto 3D models (Pelagotti et al., 2009) and for coregistration of intensity images and 3D LiDAR data (Parmehr et al., 2013).

\subsection{Direct Co-Registration of Point Clouds and Thermal In- formation}

One of the simplest approaches for directly acquiring colored 3D objects consists of using co-registered devices with known fixed 
relative orientation (Pulli et al., 1998). If the relative orientation is unknown, the standard approach consists of the manual selection of tie points and a subsequent bundle adjustment based on the collinearity equations, which has recently been used for coregistering 3D point clouds and thermal infrared images (Hoegner et al., 2014).

For automatically estimating the relative orientation between the used devices, silhouette-based approaches may be applied, which focus on minimizing the error between the contour of an object in the image and the contour of the respective projected 3D model (Lowe, 1991). In particular, linear features are often used as they typically occur in man-made environments such as urban areas. For such scenes, lines can be extracted at edges in 2D imagery, and clusters of vertical and horizontal lines can be detected in the respective $3 \mathrm{D}$ point cloud. This allows a registration based on the matching of corresponding 2D and 3D linear features (Liu and Stamos, 2005; Liu and Stamos, 2012).

As interesting alternative, which could potentially be applicable also for co-registering thermal information and 3D point cloud data, a recent approach focused on the mapping of iPhone images onto LiDAR point clouds (Sirmacek et al., 2013). In this work, the extraction of shape features from iPhone images is based on conducting mean shift segmentation, nonlinear smoothing and applying steerable filters in different orientations. Further exploiting the metafile, the GPS position and the looking angle of the iPhone camera are available. These are used for defining the orientation of a virtual plane. The extraction of shape features from point cloud data is based on the perpendicular projection of LiDAR points onto the derived virtual plane, which yields a representation for those façade and roof points of a building which are the closest to the virtual plane. Extracting the alpha shape around the points, sharp corners of the alpha shape may be used as discriminative features. Finally, a co-registration of image and point cloud data is conducted by estimating an affine transformation based on the extracted features.

\subsection{Direct Generation of Point Clouds from Thermal IR Im- ages}

In contrast to approaches involving range cameras, the direct generation of 3D models from thermal images via Structure-fromMotion (SfM) techniques has been proposed (Markov and Birk, 2007). Such image-based techniques may be applied for simultaneously recovering both 3D structure of the scene and the respective pose (i.e. position and orientation) of the camera (Szeliski, 2010). Furthermore, a thermal stereo system for recovering a 3D surface temperature map of the scene has been presented (Prakash et al., 2006) which is even able to estimate object depth within a dark environment. This system is based on exploiting isotherms (i.e. lines connecting points of equal temperature) and epipolar geometry (Hartley and Zisserman, 2008), whereby the epipolar constraints reduce the correspondence search space to the intersecting points between epipolar lines and isotherms.

\section{METHODOLOGY}

For thermal 3D mapping, we propose a methodology which automatically recovers the relative orientation between a range camera and a thermal camera (Figure 1). After conducting a radiometric correction (Section 3.1), a geometric calibration (Section 3.2 ) is applied in order to obtain undistorted 2D imagery. Subsequently, a shape-based technique for detecting feature correspondences between intensity images and thermal infrared images is exploited (Section 3.3) and, due to the acquired range information, each $2 \mathrm{D} / 2 \mathrm{D}$ correspondence can directly be assigned a respective $3 \mathrm{D}$ point which yields $3 \mathrm{D} / 2 \mathrm{D}$ correspondences. Those correspondences containing unreliable range information are discarded via an image-based rejection strategy. The remaining correspondences can efficiently be used for co-registering 3D point cloud data and thermal information (Section 3.4), i.e. mapping the thermal information onto the $3 \mathrm{D}$ point cloud.

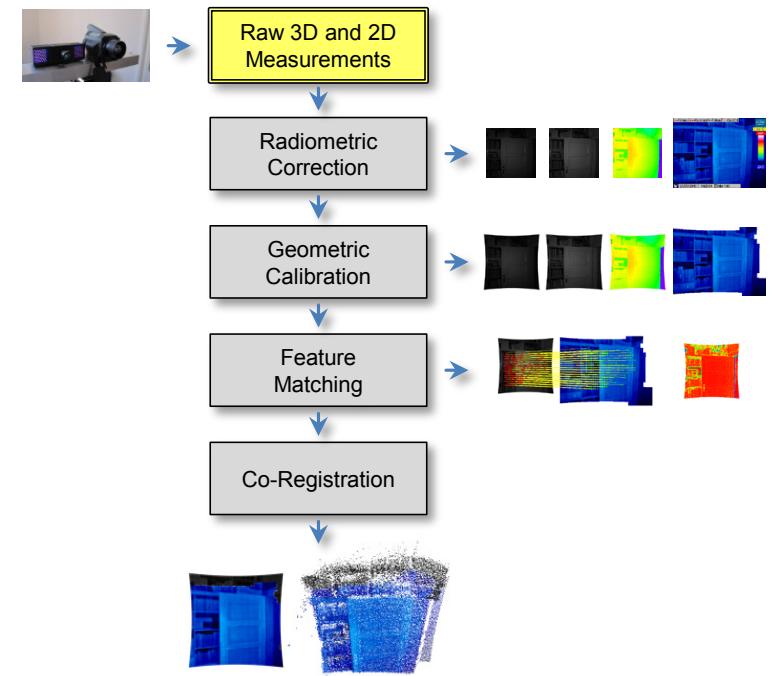

Figure 1: The components of our methodology.

\subsection{Radiometric Correction}

For range cameras, the captured intensity information corresponds to the energy of the laser light reaching the device. Due to internal processes such as conversion to a digital signal and signal amplification (which are not identical for different range cameras), this intensity information has to be adapted for applying standard image processing techniques. This is done by applying a histogram normalization of the form

$$
I_{n}=\frac{I-I_{\min }}{I_{\max }-I_{\min }} \cdot 255
$$

which adapts the intensity information $I$ of each pixel to the interval $[0,255]$ and thus yields gray-valued images. Here, the minimal and maximal intensity within all intensity measurements on the regular 2D grid are denoted as $I_{\min }$ and $I_{\max }$, respectively. The histogram normalization can be conducted for both active and passive intensity images. For the example of an indoor scene, the recorded information is visualized in Figure 2.

For thermal cameras, there is no need to apply a histogram normalization as the captured thermal information of each pixel is already assigned a color value according to a certain colorbar. A respective visualization of thermal information for the provided example is depicted in Figure 3.

\subsection{Geometric Calibration}

For calibration, we follow a recent strategy focusing on the generation of infrared-textured 3D models (Weinmann and Jutzi, 2012). This strategy involves a geometric calibration for both range camera and thermal camera.

When using range cameras, it has to be taken into account that the captured range and intensity images are distorted (Figure 2). Consequently, a camera calibration has to be carried out for such 

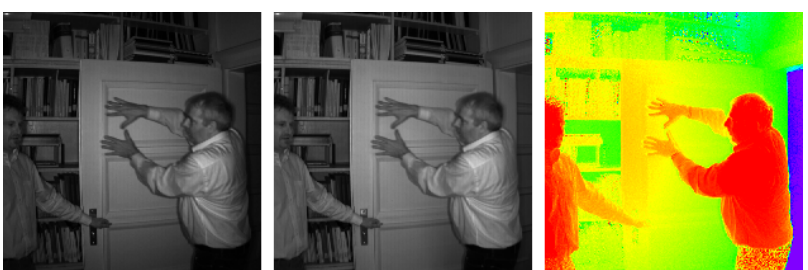

Figure 2: Visualization of the data captured with a PMD[vision] CamCube 2.0: Normalized active intensity image, normalized passive intensity image and range image (from left to right). The range is encoded in terms of a color scale reaching from red (near) via yellow, green, cyan and blue to violet (far).

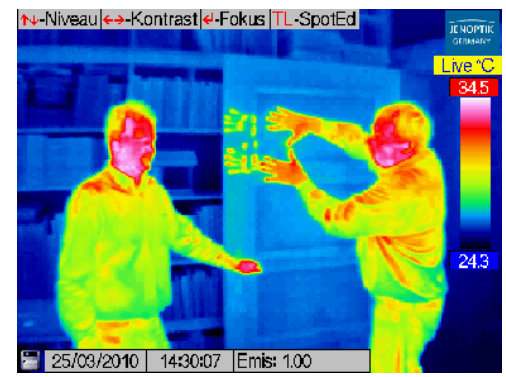

Figure 3: Visualization of thermal information captured with an InfraTec VarioCAM hr.

devices. For this purpose, we assume that the geometric mapping of a scene onto the image plane can be described with a standard camera model representing the intrinsic behavior of a digital camera. This standard model considers both radial and tangential distortion (Brown, 1971; Heikkilä and Silvén, 1997). Accordingly, the geometric mapping can be parameterized with the focal lengths in $x$ - and $y$-direction, the image coordinates $\left(x_{0}, y_{0}\right)$ of the principal point, a skew coefficient $s$, and the image distortion coefficients describing radial and tangential distortion. The well-known standard methodology then consists of using a rectangular checkerboard pattern with known size, capturing respective intensity images with the device, extracting the grid corners of the checkerboard pattern in the intensity images, and finally applying the calibration procedure (Bouguet, 2010). For the example depicted in Figure 2, the derived undistorted images are provided in Figure 4. Subsequently, the respective 3D information in the local coordinate frame can be derived (Weinmann and Jutzi, 2012).
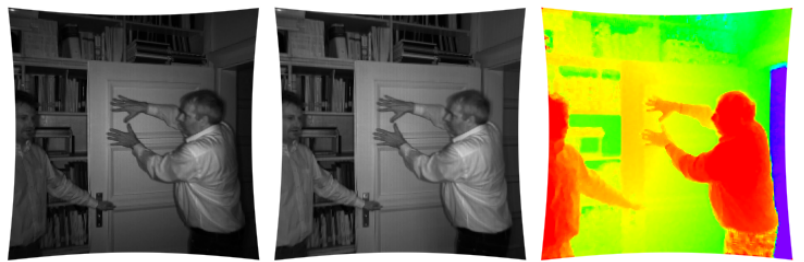

Figure 4: Visualization of the undistorted images for normalized active intensity, normalized passive intensity and range information (from left to right).

Since the checkerboard pattern is not visible in the thermal infrared domain, the calibration of thermal cameras is based on a planar testfield with lamps (Luhmann et al., 2010; Lagüela et al., 2011a; Borrmann et al., 2012b; Hoegner et al., 2014). The lamps are clearly visible in the thermal infrared images (Figure 5) and can thus easily be detected. Using a regular grid of lamps also allows for using the aforementioned standard calibration procedure for digital cameras (Figure 6).

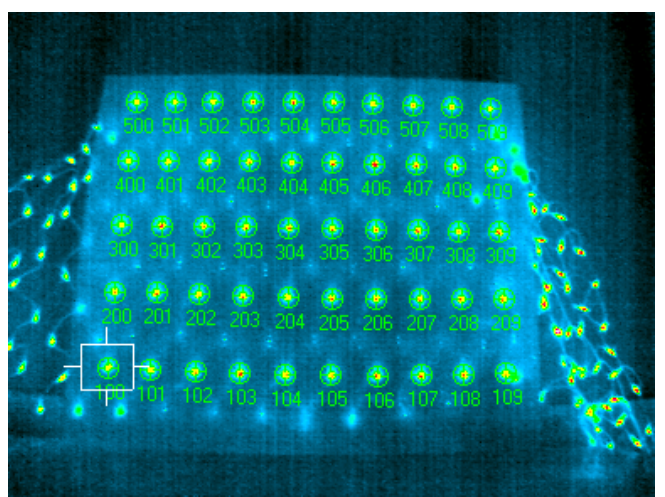

Figure 5: Captured thermal information for the planar testfield with lamps. The distortion of the thermal infrared image is clearly visible.

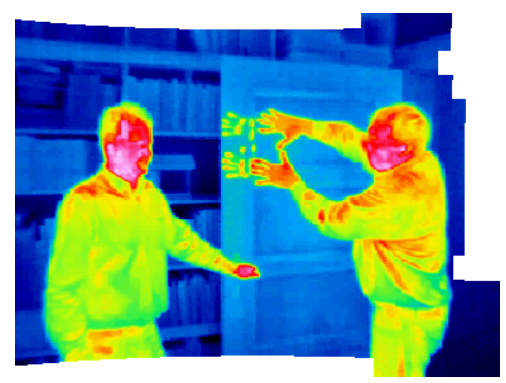

Figure 6: Visualization of the undistorted thermal infrared image.

\subsection{Detection of $2 \mathrm{D} / 2 \mathrm{D}$ and $3 \mathrm{D} / 2 \mathrm{D}$ Correspondences}

For deriving feature correspondences between the image representations of intensity and thermal infrared information, we use local features in terms of keypoints with characteristic descriptors. Due to the different characteristics of the compared images, commonly used keypoint descriptors obtained from algorithms such as SIFT (Lowe, 1999; Lowe, 2004) or SURF (Bay et al., 2011) fail in the automatic detection of point correspondences. Hence, we apply the shape-based matching algorithm proposed in (Steger, 2001) and (Ulrich, 2003) which matches image patches of a user-defined size by exploiting wavelength independent properties in terms of shape information. Thereby, the surrounding of a keypoint is described by a generated model. Thus, the algorithm is able to derive feature correspondences of which a certain percentage provides a high reliability.

In our case, a model image is generated by selecting quadratic areas of $100 \times 100$ pixels around points on a discrete grid with a spacing of 10 pixels in the intensity image provided by the range camera. The values for the size of the model image and the grid spacing were selected empirically (Weinmann et al., 2012). Subsequently, a Sobel filter is applied to the model image (Figure 7, left), and the associated gradient directions are determined for pixels with high gradient magnitude (Figure 7, center). Finally, the model image is matched to the gradients of the search image - which is represented by the thermal infrared image - by comparing the respective gradient directions (Figure 7, right). More specifically, a similarity measure is calculated which represents the normalized dot product of vector representations for the gradient directions of the transformed model and the search image (Ulrich, 2003), according to which a score can be obtained for each pixel in the search image. This similarity measure is robust in case of noise, illumination changes and partial occlusions, but not in case of changes in rotation and scale. Hence, the search 
space is extended to a predefined range of rotations and scales. If the derived similarity measure is above a certain threshold, a point correspondence is detected (Figure 8). For each correspondence, the coordinates of the center, the rotation angle and the similarity measure itself are assigned. We use the HALCON 11 implementation (MVTec Software) of the shape-based matching algorithm for our experiments.

gradient direction vectors $\boldsymbol{d}^{s}(x, y)$ of search image

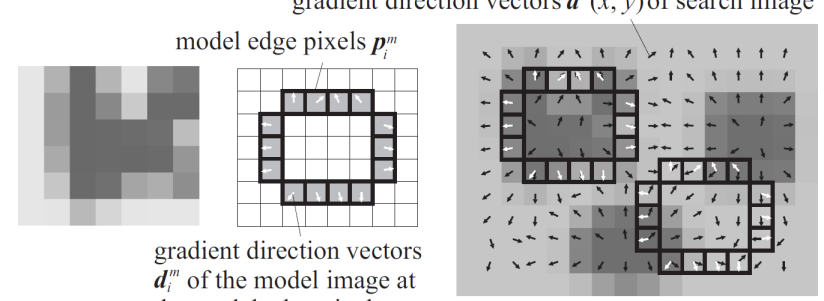

the model edge pixels

Figure 7: Principle of shape-based matching (Ulrich, 2003): model image (left), model edges (center) and search image (right).
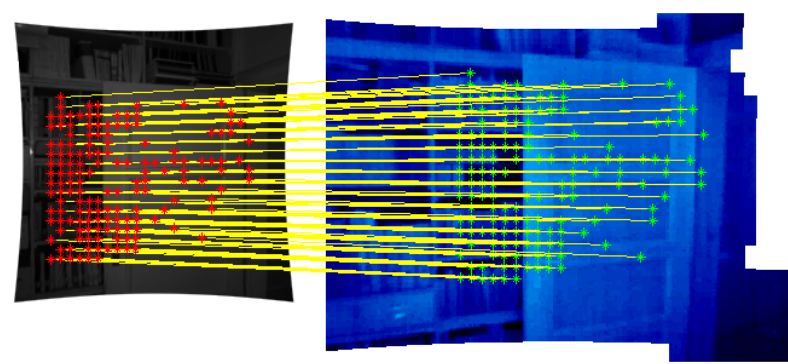

Figure 8: Automatically detected feature correspondences between an intensity image and a thermal infrared image for a static scene.

Since the range camera also provides range information in terms of a range image, we can easily assign each of the derived 2D/2D correspondences a respective $3 \mathrm{D}$ scene point whose coordinates are known in the local coordinate frame of the range camera. For estimating the relative orientation between the two utilized devices, we do not want to make strong assumptions on the scene structure, but rather exploit a general solution which relies on $3 \mathrm{D} / 2 \mathrm{D}$ correspondences, where the $3 \mathrm{D}$ information is derived via the range camera and the $2 \mathrm{D}$ information is derived via the thermal camera. Depending on the surface properties of the respective objects, i.e. shape and reflectivity, the range camera provides more or less noisy range information. Note that many points which arise from objects in the scene will probably provide a smooth surface. However, range information of points along edges of the respective objects might be very noisy. In order to detect such noisy measurements, the standard deviation $\sigma$ of all range values within a $3 \times 3$ neighborhood is calculated for each point on the regular 2D grid and used as a measure describing the reliability of the respective range information (Weinmann and Jutzi, 2011). Combining these constraints for all points on the 2D grid yields a 2D confidence map which is illustrated in Figure 9 for two exemplary scenes.

In order to remove unreliable feature correspondences, a simple thresholding is conducted. If the parameter $\sigma$ of the respective range values is larger than a predefined threshold $t_{\sigma}$, the range information of the center pixel is assumed to be unreliable, otherwise the range information of the center pixel is assumed to be reliable. Following (Weinmann and Jutzi, 2012), we select this threshold to $t_{\sigma}=0.05 \mathrm{~m}$.
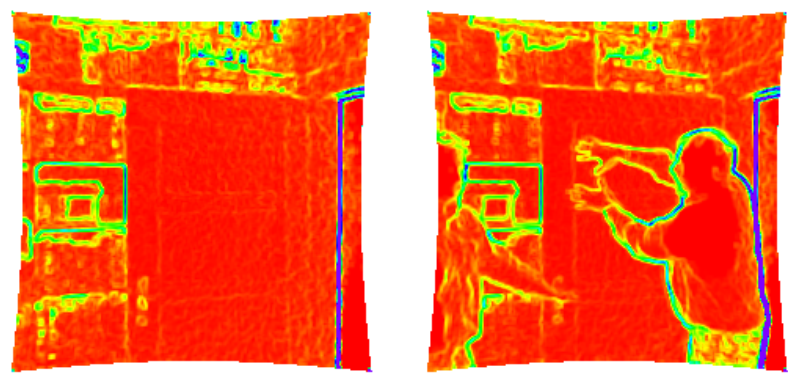

Figure 9: Confidence maps indicating the reliability of the respective range information. The reliability is encoded in terms of a color scale reaching from red (reliable) via yellow, green, cyan and blue to violet (unreliable).

\subsection{Co-Registration of 3D and 2D Information}

Once 3D/2D correspondences have been detected, the task of coregistering $3 \mathrm{D}$ and $2 \mathrm{D}$ information may be related to the wellknown Perspective- $n$-Point $(\mathrm{P} n \mathrm{P})$ problem where the aim is to estimate the exterior orientation or pose of a camera from a set of $n$ correspondences between 3D points $\mathbf{X}_{i}$ of a scene and their $2 \mathrm{D}$ projections $\mathbf{x}_{i}$ onto the image plane, where $i=1, \ldots, n$. In recent years, the Efficient Perspective- $n$-Point $(\mathrm{EP} n \mathrm{P})$ algorithm (Moreno-Noguer et al., 2007) has been proposed as a noniterative method which provides an accurate solution to the $\mathrm{P} n \mathrm{P}$ problem with only linear complexity. Compared to other approaches for solving the $\mathrm{P} n \mathrm{P}$ problem, this algorithm is not only fast and accurate, but also designed to work with a large number of correspondences and it does not require an initial estimate.

The EP $n \mathrm{P}$ algorithm is based on the idea of expressing the $n$ known 3D scene points $\mathbf{X}_{i}$ as a weighted sum of four virtual and non-coplanar control points $\mathbf{C}_{j}$ for general configurations. Denoting the weights as $\alpha_{i j}$ and introducing a superscript $c$ which indicates coordinates in the camera coordinate frame, each $3 \mathrm{D} / 2 \mathrm{D}$ correspondence provides a relation of the form

$$
w_{i}\left[\begin{array}{c}
\mathbf{x}_{i} \\
1
\end{array}\right]=\mathbf{K} \mathbf{X}_{i}^{c}=\mathbf{K} \sum_{j=1}^{4} \alpha_{i j} \mathbf{C}_{j}^{c}
$$

where $\mathbf{K}$ describes the calibration matrix. Considering the respective three equations, the scalar projective parameters $w_{i}$ can be determined according to the third equation and substituted into the other two equations. Concatenating the two modified equations for $i=1, \ldots, n$ yields a linear equation system $\mathbf{M} \mathbf{x}=\mathbf{0}$, where $\mathbf{x}$ contains the $3 \mathrm{D}$ coordinates of the four control points $\mathbf{C}_{j}$. For more details on the efficient solution of this equation system, we refer to the respective paper (Moreno-Noguer et al., 2007). Once both world coordinates and camera coordinates of the $3 \mathrm{D}$ points are known, the transformation parameters aligning both coordinate systems can be retrieved via standard methods involving a closed-form solution in the least-squares sense (Horn et al., 1988; Arun et al., 1987; Umeyama, 1991).

For a robust estimation in case of existing outlier correspondences, the RANSAC algorithm (Fischler and Bolles, 1981) represents the method of choice as it completely eliminates the influence of outlier correspondences which are not in accordance with the largest consensus set supporting the given transformation model (i.e. a 3D rigid-body transformation). Following the original implementation (Moreno-Noguer et al., 2007), the RANSAC-based $\mathrm{EP} n \mathrm{P}$ scheme relies on selecting small, but not minimal subsets of seven correspondences for estimating the model parameters and checking the whole set of correspondences for consistent 
samples. In comparison to minimal subsets, this further reduces the sensitivity to noise. In order to avoid testing all possible subsets, which would be very time-consuming, we exploit an efficient variant, where the number of iterations - which equals the number of randomly chosen subsets - is selected high enough, so that a subset including only inlier correspondences is selected with a certain probability $p$ (Hartley and Zisserman, 2008).

In our case, we may assume that the local coordinate frame of the range camera represents the reference coordinate frame. Consequently, the derived 3D coordinates are known with respect to the reference frame. Together with the respective observed 2D image locations in the thermal infrared image, they form the required 3D/2D correspondences. Note that, for this reason, the matrix $\mathbf{K}$ in Equation (2) refers to the thermal camera.

\section{EXPERIMENTAL SETUP}

For thermal 3D mapping, we use a sensor platform equipped with a range camera and a thermal camera (Figure 10), where a fixed relative orientation between the two devices is preserved.

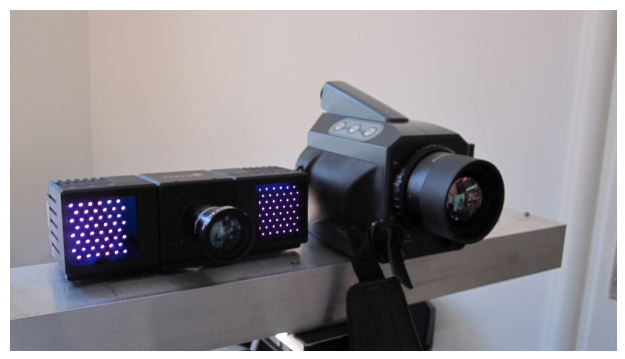

Figure 10: Sensor platform equipped with a range camera (left) and a thermal camera (right).

\subsection{Range Camera: PMD[vision] CamCube 2.0}

Modern range cameras capture geometric information as well as radiometric information and thus complementary types of data in form of images, where the information is acquired simultaneously for all pixels on the regular 2D grid. Due to the high frame rates of more than 25 releases per second, range cameras also allow to capture dynamic scenes. For our experiments, the choice of the utilized device is motivated by a possible use in outdoor environments, where range cameras based on the use of structured light are not applicable. We hence use a Time-of-Flight camera of type PMD[vision] CamCube 2.0, which measures three features for each pixel: range, active intensity and passive intensity. Note that the active intensity depends on the illumination emitted by the sensor, whereas the passive intensity depends on the background illumination arising from the sun or other external light sources. The resulting images have a size of $204 \times 204$ pixels which corresponds to a field-of-view of $40^{\circ} \times 40^{\circ}$. This in turn represents an angular resolution of approximately $0.2^{\circ}$.

Due to the measurement principle of such Time-of-Flight cameras, the non-ambiguous range depends on the modulation frequency. A modulation frequency of $20 \mathrm{MHz}$ for instance corresponds to a non-ambiguous range of $7.5 \mathrm{~m}$. In order to overcome this range measurement restriction, image- or hardware-based unwrapping procedures have recently been introduced (Jutzi, 2009; Jutzi, 2012).

\subsection{Thermal Camera: InfraTec VarioCAM hr}

As thermal camera, we use a bolometer-based InfraTec VarioCAM hr which records in the wavelength interval from $7.5-14 \mu \mathrm{m}$
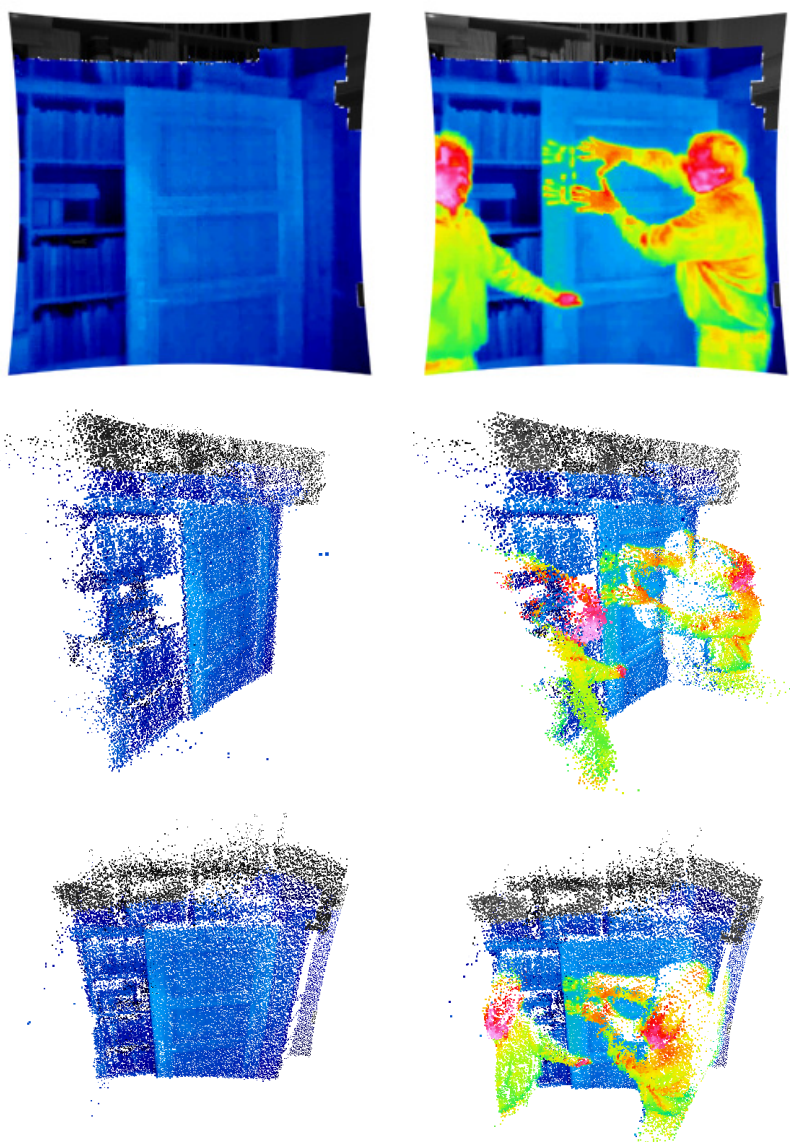

Figure 11: Visualization of thermal information mapped onto the respective $3 \mathrm{D}$ point cloud for two different scenes: $2 \mathrm{D}$ image projections (top) and colored 3D point clouds (center and bottom).

with a radiometric resolution of $0.05 \mathrm{~K}$. The captured thermal information is represented as images with a size of $384 \times 288$ pixels. The angular resolution is approximately $0.16^{\circ}$, and thus a captured thermal infrared image corresponds to a field-of-view of $61^{\circ} \times 46^{\circ}$. As the frame rate is $25 \mathrm{fps}$, this device can also be applied for observing dynamic scenes.

\section{EXPERIMENTAL RESULTS}

For testing the proposed methodology, we only have to estimate the relative orientation between the utilized devices once, which is done for the example of a static scene (Figure 1). Afterwards, co-registered data can be recorded continuously when using a (hardware or software) trigger for synchronization, since the relative orientation between the utilized devices is known.

When using the sensor platform shown in Figure 10 for observing the static indoor scene, we obtain a total number of 151 point correspondences via shape-based matching as shown in Figure 8. Of these, 14 point correspondences are discarded since the respective range information is considered to be unreliable according to the applied rejection strategy (Figure 9, left). The remaining point correspondences are exploited by the RANSAC-based $\mathrm{EP} n \mathrm{P}$ scheme, and the results in terms of thermal $3 \mathrm{D}$ mapping are shown in Figure 11 (left). Once the relative orientation is known, we can also conduct thermal 3D mapping by exploiting the known transformation parameters for other, possibly also dynamic scenes, e.g. as shown in Figure 11 (right). 


\section{DISCUSSION}

The derived results (Figure 11) clearly reveal that the presented methodology is well-suited for thermal 3D mapping. In particular, the quality is rather high due to a robust mapping between image data with different characteristics, where standard approaches typically tend to fail in finding reliable feature correspondences. Only a few pixels at the edges between the right person and the background are observable as being erroneous.

Without making strong assumptions on the 3D scene structure and without human interaction for selecting corresponding features, our proposed methodology is capable to fully automatically perform thermal 3D mapping in dynamic scenes. Thus, the proposed methodology outperforms other recent approaches which, in turn, reveal limitations as they partially rely on human interaction (Hoegner et al., 2014) or as they are only tailored for planar scene structures (Weinmann et al., 2012). Note that - without loss of generality - the methodology could also be applied for co-registering 3D point cloud data and color information, where the latter is acquired with a digital camera. This may even be significantly easier since the contours in both intensity images of the range camera and color images of the digital camera are not as blurry as in thermal infrared images.

For the sake of clarity, we also want to demonstrate that the proposed methodology for thermal 3D mapping for instance allows to observe the evolution of a $3 \mathrm{D}$ scene over time. Considering the two scenes depicted in Figure 11 (center and bottom), the respective projections onto the image plane of the range camera (Figure 11, top) and further involving the undistorted intensity and range images acquired with the range camera (e.g. Figure 4) allows a categorization of (i) background where no significant changes with respect to intensity, range or thermal information are observed, (ii) people in the scene which can be observed from a change in intensity, range and thermal information, (iii) passive scene manipulation which is indicated only by a change of intensity information, and (iv) active scene manipulation caused by interactions between people and scene objects which is indicated only by a change of thermal information. Note that already the exploitation of thresholded difference images and a connection of logical operations allows a respective statement on change detection (Figure 12). From the respective rule-based classification results according to the four considered classes of changes (Figure 13), a small error in the co-registration becomes visible which can also be observed in Figure 11. Accordingly, a respective segmentation of people in the $3 \mathrm{D}$ point cloud becomes trivial.
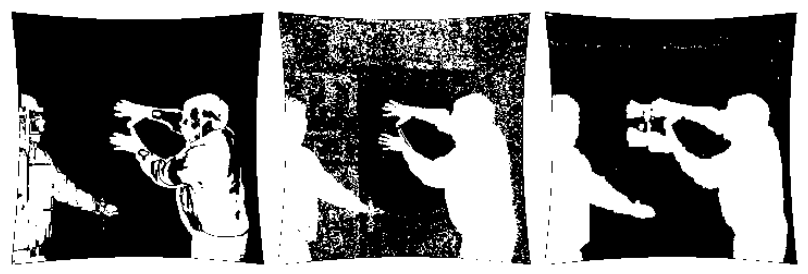

Figure 12: Thresholded difference images for intensity, range and thermal information (from left to right). Changes are indicated in white. Note the noisy behavior of range measurements.

\section{CONCLUSIONS}

In this paper, we focused on thermal 3D mapping as an important prerequisite for object detection in dynamic scenes. For this purpose, we presented a fully automatic methodology which involves (i) a radiometric correction, (ii) a common geometric calibration procedure for both range camera and thermal camera, (iii)

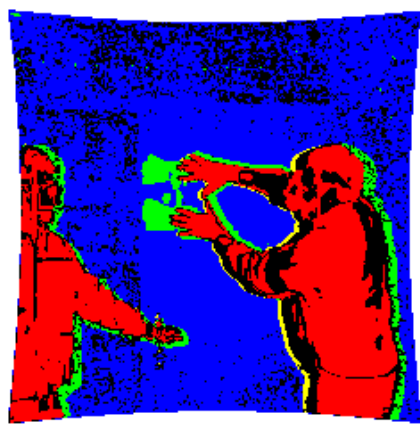

Figure 13: Results of rule-based classification based on logical operators: a clear distinction between background (blue), people (red), passive scene manipulation (yellow) and active scene manipulation (green) is possible. Note that passive and active scene manipulation are also detected at edges which indicates a small error in the co-registration.

a robust approach for detecting reliable feature correspondences between different image domains by exploiting wavelength independent properties as well as an image-based rejection strategy, and (iv) a co-registration of 3D point cloud data and thermal information based on an efficient and robust technique designed to work with a large number of $3 \mathrm{D} / 2 \mathrm{D}$ correspondences. We have demonstrated that our methodology outperforms other recent approaches in terms of both applicability and accuracy, due to avoiding both human interaction and strong assumptions on the 3D scene structure. We have also provided an example for a possible use in order to observe the evolution of a 3D scene over time which can easily be conducted by involving straightforward techniques in terms of thresholding followed by rule-based classification.

For future research, it would be desirable to not only observe a static or dynamic scene with devices mounted on a static sensor platform, but also move the sensor platform in order to capture 3D environments of larger scale and extract complete 3D models. This could for instance be valuable for building diagnostics in both indoor and outdoor environments.

\section{REFERENCES}

Alba, M. I., Barazzetti, L., Scaioni, M., Rosina, E. and Previtali, M., 2011. Mapping infrared data on terrestrial laser scanning 3D models of buildings. Remote Sensing 3(9), pp. 1847-1870.

Arun, K. S., Huang, T. S. and Blostein, S. D., 1987. Least-squares fitting of two 3-D point sets. IEEE Transactions on Pattern Analysis and Machine Intelligence 9(5), pp. 698-700.

Bai, X., Zhou, F. and Xue, B., 2011. Fusion of infrared and visual images through region extraction by using multi scale center-surround top-hat transform. Optics Express 19(9), pp. 8444-8457.

Balaras, C. A. and Argiriou, A. A., 2002. Infrared thermography for building diagnostics. Energy and Buildings 34(2), pp. 171-183.

Bay, H., Tuytelaars, T. and Van Gool, L., 2011. SURF: speeded up robust features. In: Leonardis, A., Bischof, H. and Pinz, A. (Eds.), ECCV 2006, Part I. Lecture Notes in Computer Science, Vol. 3951, Springer, Heidelberg, Germany, pp. 404-417.

Borrmann, D., Afzal, H., Elseberg, J. and Nüchter, A., 2012a. Mutual calibration for 3D thermal mapping. Proceedings of the 10th International IFAC Symposium on Robot Control.

Borrmann, D., Elseberg, J. and Nüchter, A., 2012b. Thermal 3D mapping of building façades. Proceedings of the 12th International Conference on Intelligent Autonomous Systems, pp. 173-182.

Bouguet, J.-Y., 2010. Camera calibration toolbox for Matlab [online]. Computer Vision Research Group, Department of Electrical Engineering, California Institute of Technology, Pasadena, USA. http://www.vision.caltech.edu/bouguetj/calib_doc/index.html (Accessed: 30 May 2014). 
Brown, D. C., 1971. Close-range camera calibration. Photogrammetric Engineering 37(8), pp. 855-866.

Chen, S. and Leung, H., 2009. An EM-CI based approach to fusion of IR and visual images. Proceedings of the 12th International Conference on Information Fusion, pp. 1325-1330.

Coiras, E., Santamaría, J. and Miravet, C., 2000. A segment-based registration technique for visual-IR images. Optical Engineering 39, pp. 282289.

Fischler, M. A. and Bolles, R. C., 1981. Random sample consensus: a paradigm for model fitting with applications to image analysis and automated cartography. Communications of the ACM 24(6), pp. 381-395.

Hartley, R. I. and Zisserman, A., 2008. Multiple view geometry in computer vision. University Press, Cambridge, UK.

Heikkilä, J. and Silvén, O., 1997. A four-step camera calibration procedure with implicit image correction. Proceedings of the IEEE Computer Society Conference on Computer Vision and Pattern Recognition, pp. 1106-1112.

Hoegner, L., Kumke, H., Meng, L. and Stilla, U., 2007a. Automatic extraction of textures from infrared image sequences and database integration for 3D building models. PFG Photogrammetrie, Fernerkundung, Geoinformation 6 / 2007, pp. 459-468.

Hoegner, L., Kumke, H., Schwarz, A., Meng, L. and Stilla, U., 2007b. Strategies for texturing building models with low resolution infrared image sequences. The International Archives of the Photogrammetry, Remote Sensing and Spatial Information Sciences, Vol. XXXVI-5/C55.

Hoegner, L., Roth, L., Weinmann, M., Jutzi, B., Hinz, S. and Stilla, U., 2014. Fusion von Time-of-Flight-Entfernungsdaten und thermalen IRBildern. AVN - Allgemeine Vermessungs-Nachrichten 5 / 2014, pp. 192 197.

Horn, B. K. P., Hilden, H. M. and Negahdaripour, S., 1988. Closed-form solution of absolute orientation using orthonormal matrices. Journal of the Optical Society of America A 5(7), pp. 1127-1135.

Iwaszczuk, D., Hoegner, L. and Stilla, U., 2011. Detection of windows in IR building textures using masked correlation. In: Stilla, U., Rottensteiner, F., Mayer, H., Jutzi, B. and Butenuth, M. (Eds.), Photogrammetric Image Analysis, ISPRS Conference - Proceedings. Lecture Notes in Computer Science, Vol. 6952, Springer, Heidelberg, Germany, pp. 133-146.

Jutzi, B., 2009. Investigations on ambiguity unwrapping of range images. The International Archives of the Photogrammetry, Remote Sensing and Spatial Information Sciences, Vol. XXXVIII-3/W8, pp. 265-270.

Jutzi, B., 2012. Extending the range measurement capabilities of modulated range imaging devices by time-frequency multiplexing. AVN - Allgemeine Vermessungs-Nachrichten 2 / 2012, pp. 54-62.

Lagüela, S., González-Jorge, H., Armesto, J. and Arias, P., 2011a. Calibration and verification of thermographic cameras for geometric measurements. Infrared Physics \& Technology 54, pp. 92-99.

Lagüela, S., Martínez, J., Armesto, J. and Arias, P., 2011b. Energy efficiency studies through 3D laser scanning and thermographic technologies. Energy and Buildings 43, pp. 1216-1221.

Liu, L. and Stamos, I., 2005. Automatic 3D to 2D registration for the photorealistic rendering of urban scenes. Proceedings of the IEEE Computer Society Conference on Computer Vision and Pattern Recognition, Vol. 2, pp. 137-143.

Liu, L. and Stamos, I., 2012. A systematic approach for 2D-image to 3Drange registration in urban environments. Computer Vision and Image Understanding 116(1), pp. 25-37.

Liu, Z. and Laganière, R., 2007. Context enhancement through infrared vision: a modified fusion scheme. Signal, Image and Video Processing 1(4), pp. 293-301.

Lowe, D. G., 1991. Fitting parameterized three-dimensional models to images. IEEE Transactions on Pattern Analysis and Machine Intelligence 13(5), pp. 441-450.

Lowe, D. G., 1999. Object recognition from local scale-invariant features. Proceedings of the International Conference on Computer Vision, pp. $1150-1157$.

Lowe, D. G., 2004. Distinctive image features from scale-invariant keypoints. International Journal of Computer Vision 60(2), pp. 91-110.

Luhmann, T., Ohm, J., Piechel, J. and Roelfs, T., 2010. Geometric calibration of thermographic cameras. The International Archives of the Photogrammetry, Remote Sensing and Spatial Information Sciences, Vol. XXXVIII-5, pp. 411-416.
Markov, S. and Birk, A., 2007. Detecting humans in 2D thermal images by generating 3D models. In: Hertzberg, J., Beetz, M. and Englert, R. (Eds.), KI 2007: Advances in Artificial Intelligence. Lecture Notes in Artificial Intelligence, Vol. 4667, Springer, Heidelberg, Germany, pp. 293 307.

Moreno-Noguer, F., Lepetit, V. and Fua, P., 2007. Accurate non-iterative $\mathrm{O}(\mathrm{n})$ solution to the PnP problem. Proceedings of the International Conference on Computer Vision, pp. 1-8.

Park, C., Bae, K.-H., Choi, S. and Jung, J.-H., 2008. Image fusion in infrared image and visual image using normalized mutual information. Proceedings of SPIE, Vol. 6968, pp. 69681Q-1-9.

Parmehr, E. G., Fraser, C. S., Zhang, C. and Leach, J., 2013. Automatic registration of optical imagery with 3D lidar data using local combined mutual information. ISPRS Annals of the Photogrammetry, Remote Sensing and Spatial Information Sciences, Vol. II-5/W2, pp. 229-234.

Pelagotti, A., Mastio, A. D., Uccheddu, F. and Remondino, F., 2009. Automated multispectral texture mapping of 3D models. Proceedings of the 17th European Signal Processing Conference, pp. 1215-1219.

Prakash, S., Pei, Y. L. and Caelli, T., 2006. 3D mapping of surface temperature using thermal stereo. Proceedings of the 9th International Conference on Control, Automation, Robotics and Vision, pp. 1-4.

Pulli, K., Abi-Rached, H., Duchamp, T., Shapiro, L. G. and Stuetzle, W., 1998. Acquisition and visualization of colored 3D objects. Proceedings of the 14th International Conference on Pattern Recognition, pp. 11-15.

Sirmacek, B., Lindenbergh, R. C. and Menenti, M., 2013. Automatic registration of iPhone images to laser point clouds of urban structures using shape features. ISPRS Annals of the Photogrammetry, Remote Sensing and Spatial Information Sciences, Vol. II-5/W2, pp. 265-270.

Steger, C., 2001. Similarity measures for occlusion, clutter, and illumination invariant object recognition. In: Radig, B. and Florczyk, S. (Eds.), Pattern Recognition, DAGM 2001. Lecture Notes in Computer Science, Vol. 2191, Springer, Heidelberg, Germany, pp. 148-154.

Szeliski, R., 2010. Computer vision: algorithms and applications. Springer, New York, USA.

Toth, C., Ju, H. and Grejner-Brzezinska, D., 2011. Matching between different image domains. In: Stilla, U., Rottensteiner, F., Mayer, H., Jutzi, B. and Butenuth, M. (Eds.), Photogrammetric Image Analysis, ISPRS Conference - Proceedings. Lecture Notes in Computer Science, Vol. 6952, Springer, Heidelberg, Germany, pp. 37-47.

Ulrich, M., 2003. Hierarchical real-time recognition of compound objects in images. Dissertation, German Geodetic Commission (DGK), Vol. C.

Umeyama, S., 1991. Least-squares estimation of transformation parameters between two point patterns. IEEE Transactions on Pattern Analysis and Machine Intelligence 13(4), pp. 376-380.

Vidas, S., Moghadam, P. and Bosse, M., 2013. 3D thermal mapping of building interiors using an RGB-D and thermal camera. Proceedings of the IEEE International Conference on Robotics and Automation, pp. 2311-2318.

Weinmann, M., 2013. Visual features - From early concepts to modern computer vision. In: Farinella, G. M., Battiato, S. and Cipolla, R. (Eds.), Advanced Topics in Computer Vision. Advances in Computer Vision and Pattern Recognition, Springer, London, UK, pp. 1-34.

Weinmann, M. and Jutzi, B., 2011. Fully automatic image-based registration of unorganized TLS data. The International Archives of the Photogrammetry, Remote Sensing and Spatial Information Sciences, Vol. XXXVIII-5/W12, pp. 55-60.

Weinmann, M. and Jutzi, B., 2012. A step towards dynamic scene analysis with active multi-view range imaging systems. The International Archives of the Photogrammetry, Remote Sensing and Spatial Information Sciences, Vol. XXXIX-B3, pp. 433-438.

Weinmann, M., Hoegner, L., Leitloff, J., Stilla, U., Hinz, S. and Jutzi, B., 2012. Fusing passive and active sensed images to gain infrared-textured 3D models. The International Archives of the Photogrammetry, Remote Sensing and Spatial Information Sciences, Vol. XXXIX-B1, pp. 71-76.

Xue, Z., Blum, R. S. and Li, Y., 2002. Fusion of visual and IR images for concealed weapon detection. Proceedings of the International Conference on Image Fusion, pp. 1198-1205.

Yao, F. and Sekmen, A., 2008. Multi-source airborne IR and optical image fusion and its application to target detection. Proceedings of the 4th International Symposium on Advances in Visual Computing, pp. 651-660.

Zitová, B. and Flusser, J., 2003. Image registration methods: a survey. Image and Vision Computing 21(11), pp. 977-1000. 